\title{
Proposal for real-time terahertz imaging system with palm-size terahertz camera and compact quantum cascade laser
}

Naoki Oda, Alan W. Lee, Tsutomu Ishi, Iwao Hosako, Qing $\mathrm{Hu}$

Naoki Oda, Alan W. M. Lee, Tsutomu Ishi, Iwao Hosako, Qing Hu, "Proposal for real-time terahertz imaging system with palm-size terahertz camera and compact quantum cascade laser," Proc. SPIE 8363, Terahertz Physics, Devices, and Systems VI: Advanced Applications in Industry and Defense, 83630A (9 May 2012); doi: 10.1117/12.917682

Event: SPIE Defense, Security, and Sensing, 2012, Baltimore, Maryland, United States 
Invited Paper

\title{
Proposal for Real-Time Terahertz Imaging System, with
}

\section{Palm-size Terahertz Camera and Compact Quantum Cascade Laser}

\author{
Naoki Oda ${ }^{\mathrm{a} 1}$, Alan W. M. Lee ${ }^{\mathrm{b}, \mathrm{c}}$, Tsutomu Ishi ${ }^{\mathrm{a}}$, Iwao Hosako ${ }^{\mathrm{d}}$, and Qing $\mathrm{Hu}^{\mathrm{c}}$ \\ ${ }^{a}$ NEC Guidance and Electro-Optics Division \\ ${ }^{\mathrm{b}}$ LongWave Photonics LLC \\ ${ }^{c}$ Massachusetts Institute of Technology \\ ${ }^{d}$ National Institute of Information and Communications Technology
}

\begin{abstract}
This paper describes a real-time terahertz $(\mathrm{THz})$ imaging system, using the combination of a palm-size $\mathrm{THz}$ camera with a compact quantum cascade laser (QCL). The THz camera contains a 320x240 microbolometer focal plane array which has nearly flat spectral response over a frequency range of ca. 1.5 to $100 \mathrm{THz}$, and operates at $30 \mathrm{~Hz}$ frame rate. The QCL is installed in compact cryogen-free cooler. A variety of QCLs are prepared which can cover frequency range from ca. 1.5 to $5 \mathrm{THz}$. THz images of biochemical samples will be presented, using the combined imaging system. Performance of the imaging system, such as signal-to-noise ratio of transmission-type THz microscope, is predicted.
\end{abstract}

Keywords : THz microbolometer focal plane array, Quantum Cascade Laser, Microscope

\section{INTRODUCTION}

Terahertz $(\mathrm{THz})$ technology is very much expected to be applied to biomedical imaging ${ }^{[1],[2]}$, non-destructive inspection ${ }^{[3]}$, security ${ }^{[4],[5]}$ and so forth, because biological macromolecules have spectral features in the $\mathrm{THz}$ frequency region, and THz radiation is transparent to papers, clothes, plastics, etc. Time Domain Spectroscopy (TDS) technique has been used in most of cases to investigate spectral features for a variety of materials and depth profiles of layered materials since early $2000^{[6],[7]}$. Along with spectroscopic systems, THz imaging techniques have also been developed, using TDS with raster scanning of a sample ${ }^{[8]}$. However, this technique requires long acquisition times to form an imaging, making the development of real-time $\mathrm{THz}$ imaging desirable.

Microbolometer focal plane arrays (FPAs) have recently been developed specifically for the THz frequency range by several groups ${ }^{[9]-[11]}$. NEC, the company of the first author of this paper, has begun initial production of a palm-size $\mathrm{THz}$

1 Correspondence Email: n-oda@cb.jp.nec.com Phone:+81-42-333-1150; Fax:+81-42-333-1888

Terahertz Physics, Devices, and Systems VI: Advanced Applications in Industry and Defense,

edited by A. F. Mehdi Anwar, Nibir K. Dhar, Thomas W. Crowe, Proc. of SPIE Vol. 8363, 83630A

(c) 2012 SPIE $\cdot$ CCC code: $0277-786 X / 12 / \$ 18 \cdot$ doi: $10.1117 / 12.917682$

Proc. of SPIE Vol. $836383630 \mathrm{~A}-1$ 
camera which operates at a $30 \mathrm{~Hz}$ frame rate ${ }^{[12]}$. Since microbolometer FPAs have thermal isolation structures (TIS) with typical thermal time-constants of $10-20 \mathrm{msec}$, THz radiation sources which operate in continuous wave $(\mathrm{CW})$ or in pulsed mode with high duty cycles are highly desirable. Furthermore, the sensitive frequency range of THz-FPA has to be matched to emission frequency range of the THz source. The microbolometer THz-FPA described in this paper is sensitive in the frequency range of ca. 1.5 to $100 \mathrm{THz}$. This frequency range is shown to match well with the emission frequency range of quantum cascade lasers $(\mathrm{QCLs})^{[13]-[16]}$ and THz parametric generators (TPGs) ${ }^{[17]}$. The combination of these sources and the microbolometer THz-FPAa provide the basis for a real-time imaging system. .As the microbolometer THz-FPA responds to average power, the high-power levels of QCLs make these devices a more suitable illumination source.

The active region of THz-QCLs consist of a GaAs/AlGaAs superlattice, engineered to provide gain at frequencies ranging from 1.5 to $5 \mathrm{THz}$. The optical mode is typically confined to the active region by defining a ridge, and using top and bottom metal layers, a so called metal-metal waveguide, similar to a microstrip transmission line. A benefit to the metal-metal waveguide is that it provides an efficient heat removal mechanism. This allows THz-QCLs to dissipate more power, allowing efficient operation in $\mathrm{CW}$ or pulsed mode, with the high time-average powerlevels that are beneficial for illumination of the microbolometer THz-FPA.. THz QCL devices showing the highest output power levels and operating temperatues have been developed at MIT ${ }^{[13]}$ and are currently being commercialized by LongWave Photonics LLC.

This paper first describes the characteristics of microbolometer THz-FPA with $320 \times 240$ format and $23.5 \mu \mathrm{m}$ pixel pitch and presents beam patterns for a couple of $\mathrm{THz}$ sources taken with evaluation $\mathrm{THz}$ camera. Specification of palm-size THz camera is also shown. Second, characteristics of QCL and specification of easy-to-handle QCL equipment are described and THz imageries taken with QCL and THz camera are presented. Finally, design for transmission-type $\mathrm{THz}$ microscope is explained.

\section{MICROBOLOMETER THz FOCAL PLANE ARRAY (THz-FPA)}

Two types of THz-FPAs, namely, broad-band type and narrow-band type microbolometer THz-FPAs, have been developed so far ${ }^{[9],[18],[19]}$ and they have $320 \times 240$ format and $23.5 \mu \mathrm{m}$ pixel pitch. Figure 1 shows pixel structures of both broad-band and narrow-band types. The broad-band type has thermal isolation structure (TIS). The lower part of TIS is composed of silicon ( $\mathrm{Si}$ ) read-out integrated circuit (ROIC), while the upper part of TIS is composed of diaphragm and eaves sensitive to $\mathrm{THz}$ radiation, and two legs which support the diaphragm. Cell contacts electrically connect the upper part to the Si ROIC in the lower part. The narrow-band type additionally has a Si cover above the TIS.

The diaphragm is composed of a vanadium oxide (VOx) bolometer layer, passivation layers (silicon nitride (SiNx)) and electrodes, while the eaves are composed of a SiNx layer. THz absorption layer (thin metallic film) is formed on both diaphragm and eaves. The leg is composed of electrically conductive layer and SiNx passivation layers. Reflection layer is formed at the bottom of air gap to provide an absorption mechanism for incident THz radiation. The absorption mechanism of TIS was explained in details ${ }^{[9],[18],[19]}$. In this paper, features are briefly described. 
The pixel of the broad-band type THz-FPA (Fig.1 (a)) has pseudo-optical cavity structure which is formed between $\mathrm{THz}$ absorption layer and reflector. In the case that one wants to get maximum sensitivity at a certain wavelength $\left(\lambda_{0}\right)$, the optical path length between $\mathrm{THz}$ absorption layer and reflector is set to $\lambda_{0} / 4$ and sheet resistance of $\mathrm{THz}$ absorption layer is adjusted to $377 \Omega /$ square (vacuum resistance). However, this optical cavity structure results in a sensitivity dip at a wavelength of $\lambda_{0} / 2$. To avoid the dip, the pseudo-optical cavity structure is taken with a certain range of sheet resistance for THz absorption layer ${ }^{[9],[18]}$, on the basis of pixel structure of microbolometer infrared $\mathrm{FPA}^{[20]}$.

Pixel structure of the narrow-band type microbolometer THz-FPA is shown in Fig.1 (b). The Si cover with an anti-reflection coating deposited on the incident side is put above $\mathrm{THz}$ absorption layer at a distance of half of wavelength of interest. As explained in the paper ${ }^{[19]}$, responsivity of this type THz-FPA is enhanced by a factor of 2 to 3 .

(a)

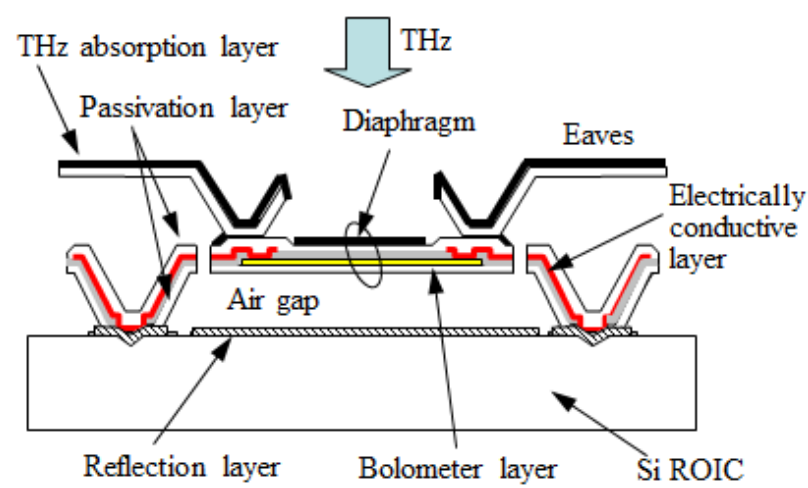

(b)

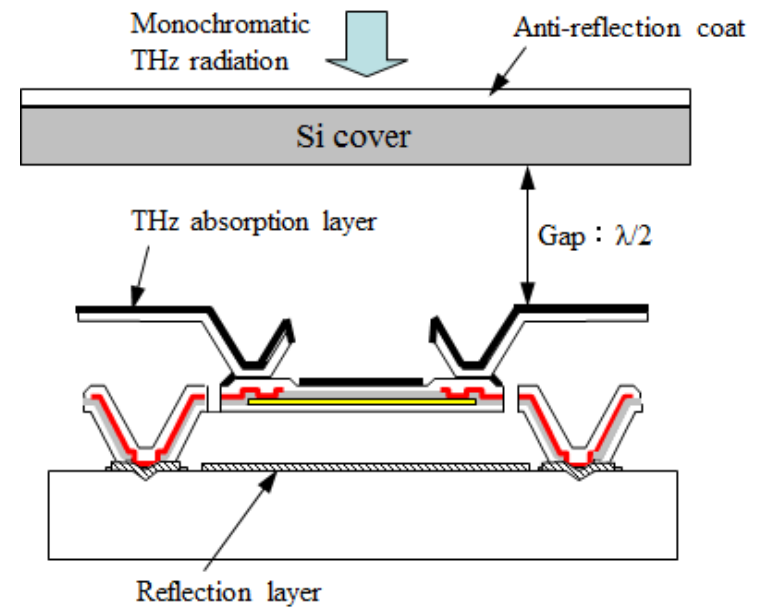

Fig.1 Pixel structures of (a) broad-band type THz-FPA and (b) narrow-band type THz-FPA

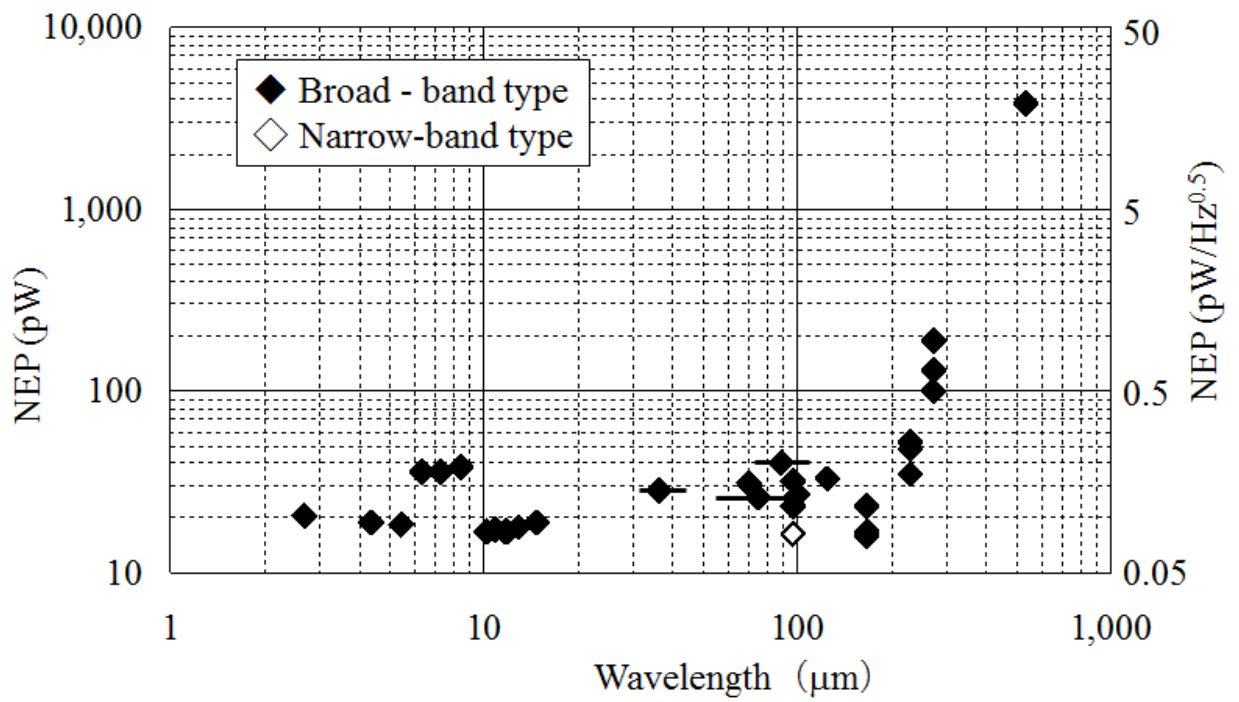

Fig. 2 Wavelength dependence of NEP 
Table 1 Measurement methods of NEP and measurement laboratories

\begin{tabular}{|c|c|c|c|}
\hline \multirow{2}{*}{$\begin{array}{l}\text { Wavelengths } \\
\qquad(\mu \mathrm{m})\end{array}$} & \multicolumn{2}{|c|}{ Measurement methods } & \multirow{2}{*}{$\begin{array}{l}\text { Measurement labs. } \\
\text { (Collaborators) }\end{array}$} \\
\hline & Radiation sources & Calibration of incident power & \\
\hline $\begin{array}{l}2.67 \mu \mathrm{m}, 4.38,5.42, \\
6.32,7.24,8.42, \\
10.24,10.89,11.74, \\
11.93,12.82,14.65\end{array}$ & \multirow[t]{4}{*}{$\begin{array}{l}\text { Combinations of } \\
\text { blackbody with filters }\end{array}$} & \multirow[t]{4}{*}{$\begin{array}{l}\text { Calculations of power, using Planck } \\
\text { function and filter transmission curves }\end{array}$} & \multirow[t]{4}{*}{ NEC } \\
\hline $31-43 \mu \mathrm{m}$ & & & \\
\hline $55-95 \mu \mathrm{m}$ & & & \\
\hline $72-107 \mu \mathrm{m}$ & & & \\
\hline $70 \mu \mathrm{m}(4.3 \mathrm{THz})$ & \multirow{3}{*}{$\begin{array}{l}\text { QCL and long pass metal } \\
\text { mesh filter (LPMMF; } \lambda \\
>33 \mu \mathrm{m})\end{array}$} & $\begin{array}{l}\text { Power meter (Scientec Inc., Model: } \\
\mathrm{AC} 2500 \mathrm{H})\end{array}$ & $\mathrm{MIT}, \mathrm{LWP}^{1)}$ \\
\hline $97 \mu \mathrm{m}(3.1 \mathrm{THz})$ & & $\begin{array}{l}\text { Power meter (Spectrum Detector Inc., } \\
\text { Model: SPI-A-62THZ) }\end{array}$ & NICT \\
\hline $125 \mu \mathrm{m}(2.4 \mathrm{THz})$ & & $\begin{array}{l}\text { Liquid helium cooled Ge bolometer with } \\
\text { calibrated responsivity }^{3)} \\
\text { (QMC Instruments, Model: QGeB/2) }\end{array}$ & $\begin{array}{l}\text { ISAE }^{2)} \\
\text { (Dr. F. Destic) }\end{array}$ \\
\hline $\begin{array}{l}100 \mu \mathrm{m}(3 \mathrm{THz}) \\
167 \mu \mathrm{m}(1.8 \mathrm{THz}) \\
231 \mu \mathrm{m}(1.3 \mathrm{THz}) \\
273 \mu \mathrm{m}(1.1 \mathrm{THz})\end{array}$ & $\begin{array}{l}\text { is-TPG and LPMMF } \\
(\lambda>33 \mu \mathrm{m})\end{array}$ & $\begin{array}{l}\text { Power meter (Spectrum Detector Inc., } \\
\text { Model: SPI-A-65 THZ) }\end{array}$ & $\begin{array}{l}\text { RIKEN } \\
\text { (Drs. H. Minamide } \\
\text { and S. Hayashi) }\end{array}$ \\
\hline $532 \mu \mathrm{m}(0.56 \mathrm{THz})$ & $\begin{array}{l}\text { Resonance tunneling } \\
\text { diode and LPMMF } \\
(\lambda>33 \mu \mathrm{m})\end{array}$ & Power meter (Erickson, Model: PM3) & $\begin{array}{l}\text { Tokyo Institute of } \\
\text { Technology (Prof. } \\
\text { M. Asada) }\end{array}$ \\
\hline
\end{tabular}

\footnotetext{
1) LongWave Photonics LLC

$\left.{ }^{2}\right)$ Institut Superieur de l'Aeronautique et de l'Espace

${ }^{3}$ ) Bolometer responsivity measured at $3.4 \mathrm{THz}$ is $11 \pm 0.9 \mathrm{kV} / \mathrm{W}$.
}

Wavelength dependence of NEP for the broad-band type THz-FPA and NEP value at $97 \mu$ m for narrow-band type THz-FPA are shown in Fig. 2, where band width of $42.7 \mathrm{kHz}$ is taken into account. Table 1 summarizes measurement methods of NEP and measurement laboratories. The measurements for the broad-band type THz-FPA were made for the same FPA chip except for wavelength range from 2.7 to $14.7 \mu \mathrm{m}$, where single element bolometers with the same structure as pixel formed around THz-FPA were used ${ }^{[9]}$. In the measurements, a variety of THz radiation sources such as blackbody, QCLs, is-TPG (injection seeded - Terahertz Parametric Generator) and resonance tunneling diode were used, 
and different calibration methods of measuring incident power were used, such as power meter manufactured by different companies. Despite the absence of standardized NEP measurement methods in the THz region, the measured NEP for the broad-band type is smoothly connected and is relatively flat over the range of 2.7 to $200 \mu \mathrm{m}$, after which it begins to degrade. This suggests that it is not difficult to establish a standardized method of measuring performance of $\mathrm{THz}$ detectors.

\section{THz IMAGERIES}

Several real-time $\mathrm{THz}$ imageries were reported $\mathrm{in}^{[9],[19]}$, such as THz image of reflected QCL beam under hot and black smoke, passive $\mathrm{THz}$ images of person, label-free detection images at $\mathrm{THz}$ for small molecule-protein reaction, and so forth. Here, broad-band type microbolometer THz-FPA (320x240 - $23.5 \mu \mathrm{m}$ pitch) is installed in THz evaluation camera which is applied to make measurements of beam patterns of $\mathrm{THz}$ radiation sources at different laboratories.

Figure 3 shows experimental setup for measuring the beam pattern of a TDS instrument (EKSPLA, http://www.ekspla.com/). The unit containing single element $\mathrm{THz}$ detector was replaced with the $\mathrm{THz}$ camera and off-axis parabolic mirror. In this setup, silicon ( $\mathrm{Si}$ ) lens of the THz camera was removed and only long pass metal mesh filter with cut-on wavelength of $33 \mu \mathrm{m}$ was attached. Circular beam-pattern with ca. $0.5 \mathrm{~mm}$ in diameter was obtained at $60 \mathrm{~Hz}$ frame rate with signal-to-noise ratio (SNR) of 3 to 4 . When frame integration (16 frames) and spatial filter (area integration over the number of pixels $32 \times 32$ ) were applied, SNR value was increased to ca. 100 . This experiment indicates that THz camera is an efficient tool for optical alignment of TDS system.

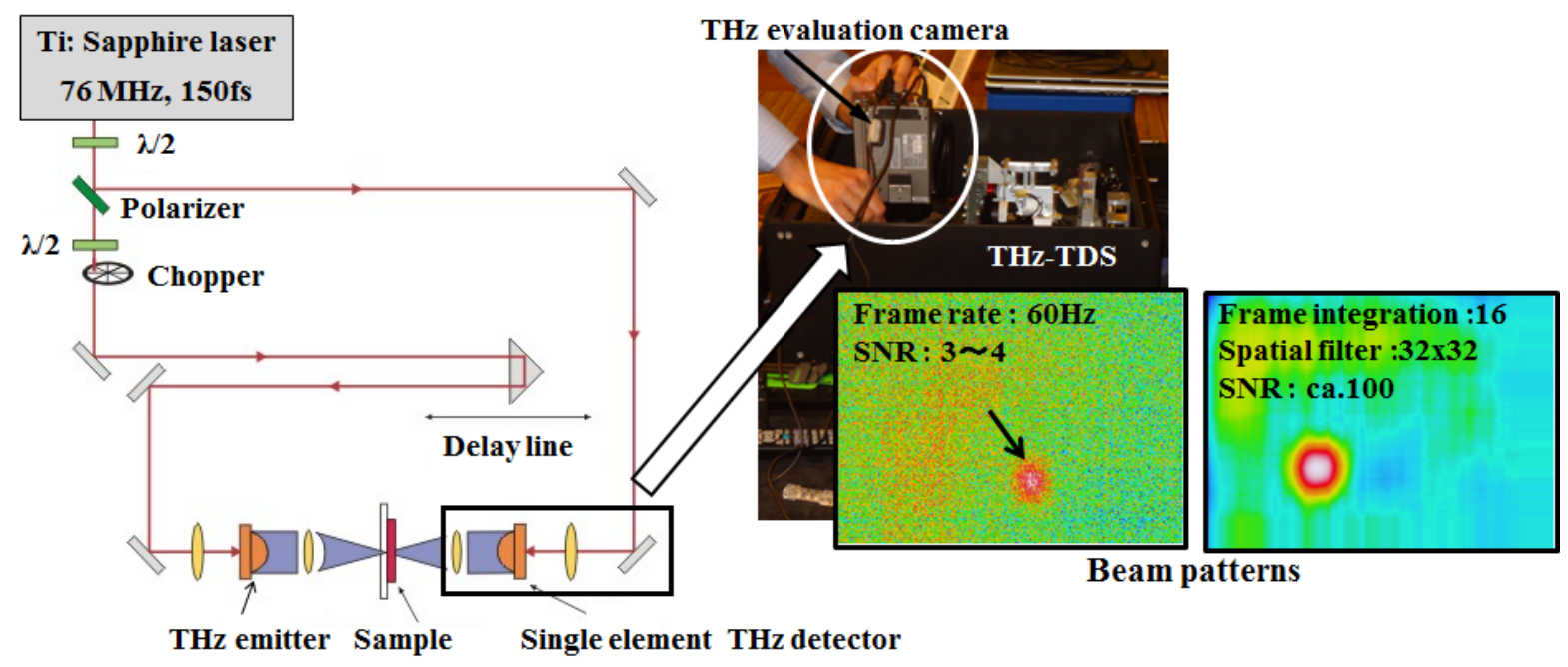

Fig.3 Beam patterns of THz-TDS instrument obtained with THz evaluation camera (in collaboration with EKSPLA at the exhibition of IRMMW-THz2011/Houston on 2011 Oct.)

Figure 4 shows a rough idea on experimental setup for measuring beam pattern of $\mathrm{THz}$ emission from air plasma. Two lasers (wavelengths of $800 \mathrm{~nm}$ and $400 \mathrm{~nm}$ ) were focused at a certain part of air. Here, the $400 \mathrm{~nm}$ laser was created by $\mathrm{BBO}$ ( $\beta$-barium borate) crystal. THz radiation emitted from air plasma was focused at THz-FPA in the THz camera 
with two off-axis parabolic mirrors (OAPs). In this setup, Si lens of the THz camera was removed, while long pass metal mesh filter with cut-on wavelength of $33 \mu \mathrm{m}$ and germanium (Ge) disk acting as attenuator were put. One can clearly see beam pattern with SNR of ca. 140. If the Ge disk was removed, SNR would be increased to 460 .

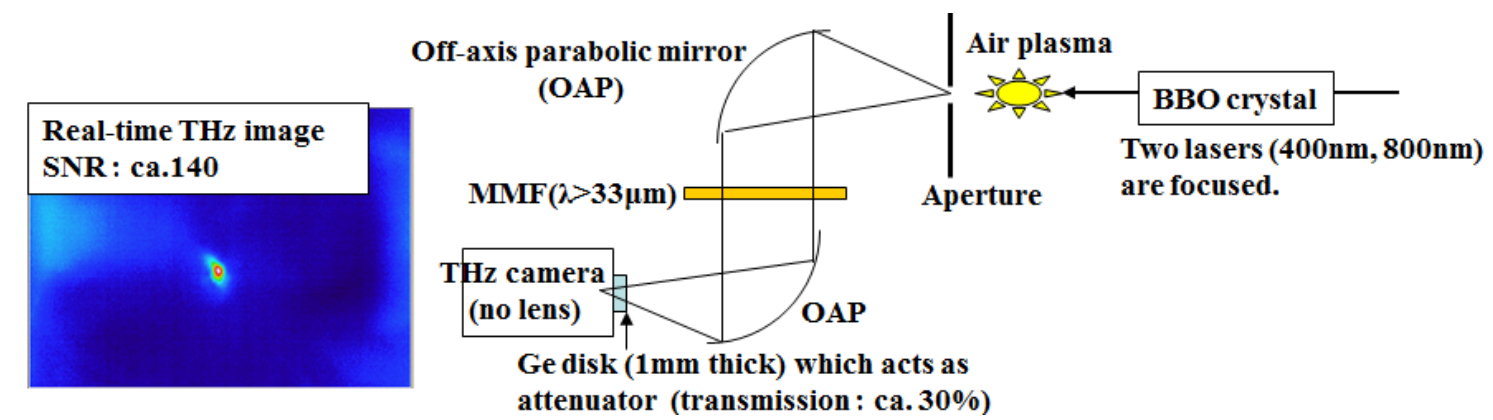

Fig.4 Beam pattern of THz emission from air plasma (in collaboration with the Institute for Solid State Physics, the Univ. of Tokyo on $2010 \mathrm{Feb}$.)

Figure 5 shows beam patterns of $\mathrm{THz}$ emission from a non-linear $\mathrm{LiNbO}_{3}$ crystal excited by tilted-pump pulse-front femtosecond laser ${ }^{[21]}$. Because of difficulty in alignment of optics, it took a couple of days to obtain $\mathrm{THz}$ signal from the non-linear optical crystal (SNR: ca. 80, the left image in Fig.5). Once THz camera was applied to this THz radiation source, adjustment of optics for 5 - 10 minutes improved SNR by a factor of 30 (the right image in Fig.5). The rightmost picture in Fig. 5 shows a real-time THz transmission image of sample which was obtained with similar THz source. The sample has a Chinese character shaped with $\mathrm{Al}$ foil on Teflon substrate. This experiment as well as other example ${ }^{[22]}$ again indicate that $\mathrm{THz}$ camera is very efficient for optical alignment in complicated $\mathrm{THz}$ radiation source.
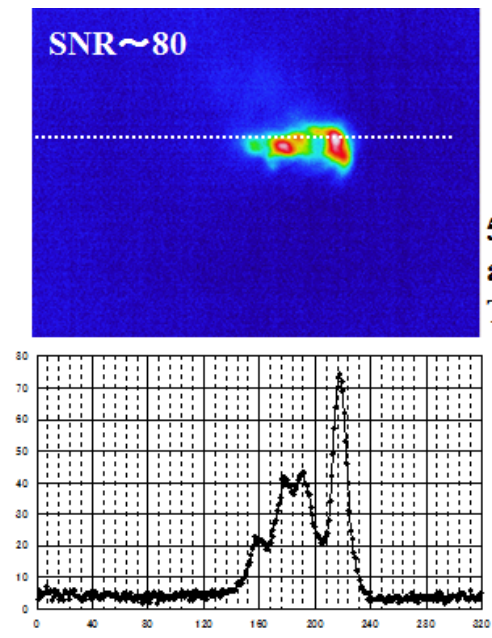
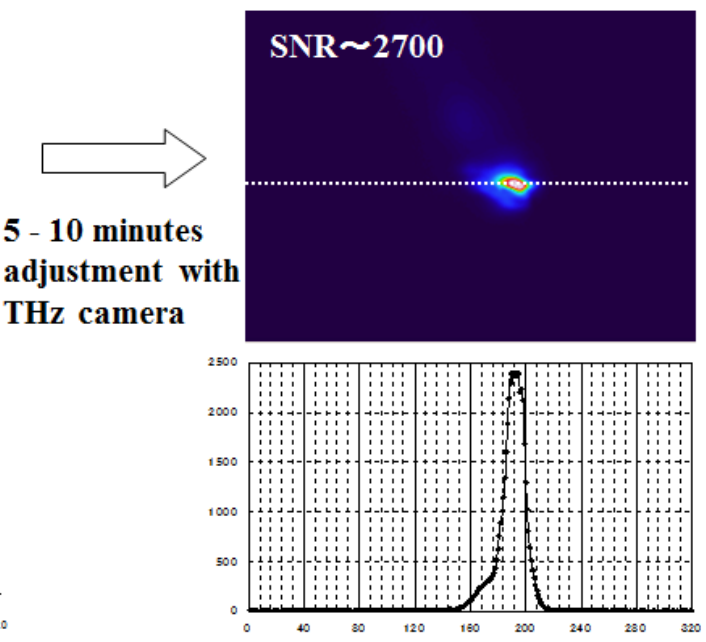

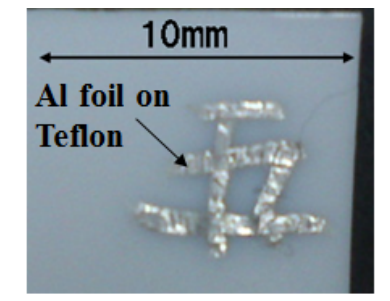

Picture of sample

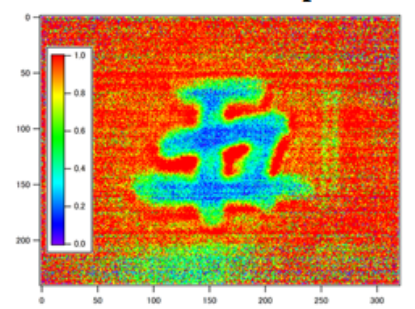

THz image of the sample

Fig.5 Beam patterns of $\mathrm{THz}$ emission from non-linear optical crystal excited by tilted-pump pulse-front femtosecond laser (in collaboration with the Univ. of Tokyo on 2010 Mar.) 
Palm-size THz camera has been developed for real-time imaging, on the basis of the THz evaluation camera used above. This camera incorporates broad-band type THz-FPA. Specification and picture of the camera are shown in table 2 and Fig.6, respectively. The new feature of lock-in imaging function is added to the previous version ${ }^{[12]}$.

Table 2. Specifications of palm-size THz camera

\begin{tabular}{|l|l|}
\hline Array format - Pixel pitch & $320 \times 240-23.5 \mu \mathrm{m}$ \\
\hline Field of view & ca. $15^{\circ} \times 12^{\circ}$ \\
\hline Outputs & $\begin{array}{l}\text { Digital image data : USB2.0 } \\
\text { Sync. signal : BNC }\end{array}$ \\
\hline New feature & Lock-in imaging function \\
\hline Weight & ca. $550 \mathrm{~g}($ excl. lens and filter $)$ \\
\hline Size & $\begin{array}{l}\text { ca. } 64 \mathrm{~mm}^{\mathrm{W}} \times 62 \mathrm{~mm}^{\mathrm{H}} \times 103 \mathrm{~mm}^{\mathrm{D}} \\
\text { (excl. lens and filter }) \\
{[D: \text { ca. } 170 \mathrm{~mm} \text { with lens and filter }]}\end{array}$ \\
\hline
\end{tabular}

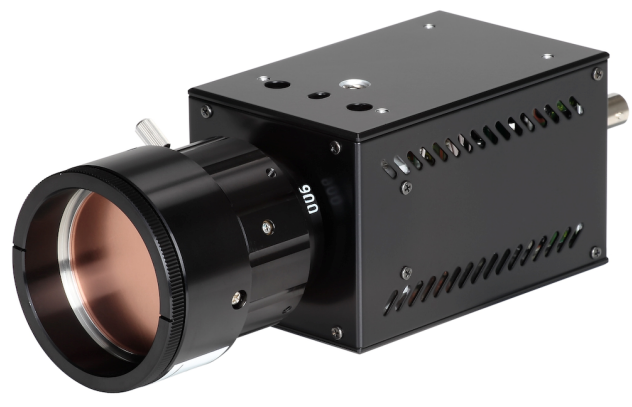

Fig.6 Palm-size THz camera

\section{QUANTUM CASCADE LASER (QCL)}

Growth, fabrication, and characterization of QCLs are detailed in reference ${ }^{[23]}$. Briefly, QCL structure was grown by molecular beam epitaxy, based on the resonant-phonon depopulation scheme ${ }^{[24]}$. The conduction band profile of a typical design (lasing frequency $3.4 \mathrm{THz}, \mathrm{E}_{54}=13.9 \mathrm{meV}$ ) is shown under electrical bias in Fig.7. An individual gain module consists of four quantum wells of GaAs/ $\mathrm{Al}_{.15} \mathrm{Ga}_{.85} \mathrm{As}$ (encircled in a dashed line). This single gain module is cascaded $\sim 180$ times to form the QCL active region. The colored lines represent the amplitude squared electron wavefunctions and are labeled 1-5. The radiative transition, namely lasing transition, occurs between level 5 and 4 . Electrons in the lower radiative level 4 scatter readily into level 3, which is spatially extended over several quantum wells, and strongly overlapping with levels 2 and 1 . This overlap allows fast non-radiative depopulation of the lower radiative state via LO-phonon scattering $\left(\hbar \omega_{\mathrm{LO}} \sim 39.3 \mathrm{meV}\right)$. Electrons are injected from level 1 back to level 5 of the next module, completing the cascade pumping scheme.

The QCL active region is fabricated into metal-metal waveguides (MM waveguide, Fig.7), using semiconductor fabrication techniques. This waveguide consists of a ground plane, on which the GaAs/AlGaAs active region is lithographically defined, with a top metal contact. Typical dimensions for this waveguide are $1 \mathrm{~mm} \times 100 \mu \mathrm{m} \times 10 \mu \mathrm{m}$. This waveguide allows near unity optical mode overlap with the gain medium, leading to lower laser threshold-currents and higher operating temperature performance. However, this high confinement also results in high end-facet reflectivities of $70 \%$ to $90 \%$ depending on the wavelength and geometry. To increase the outcoupling (reduce the reflectivity), a silicon micro-lens is attached to the facet of the waveguide (left in Fig.8). This lens has two very desirable effects: increasing the output power by factors of $\sim 5$, and dramatically reducing the beam divergence to values $<30 \mathrm{deg}$. After the lens is attached, the device is packaged (right picture in Fig.8). 

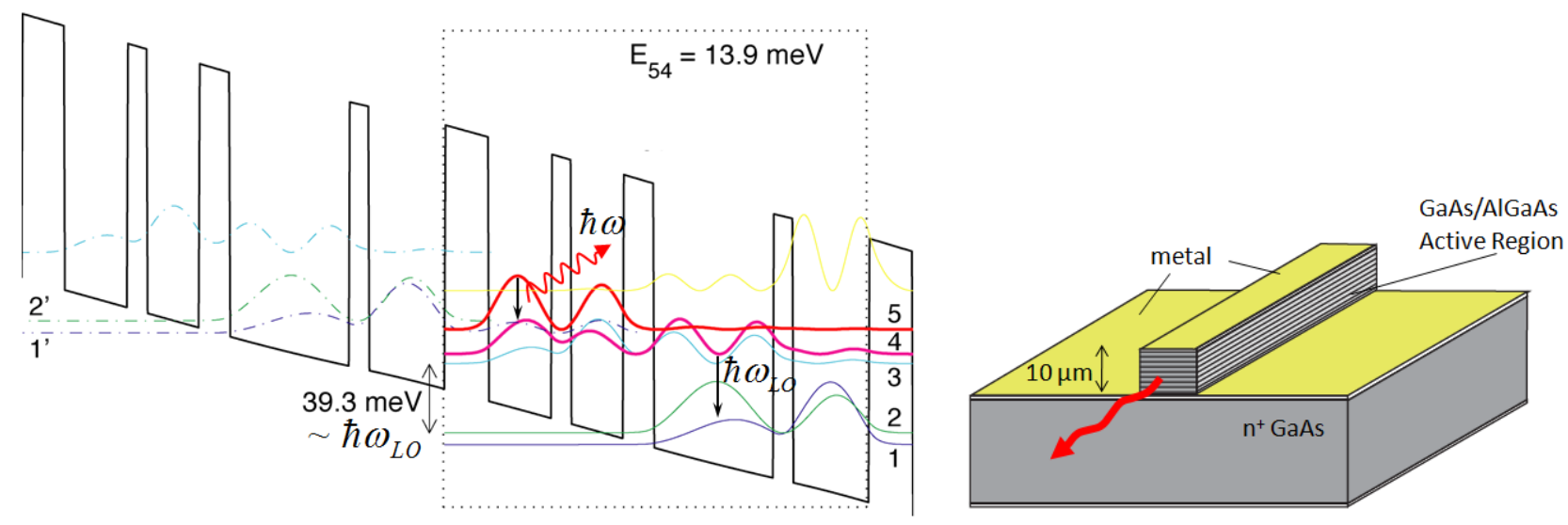

Fig.7 Band structure of QCL and metal-metal waveguide structure
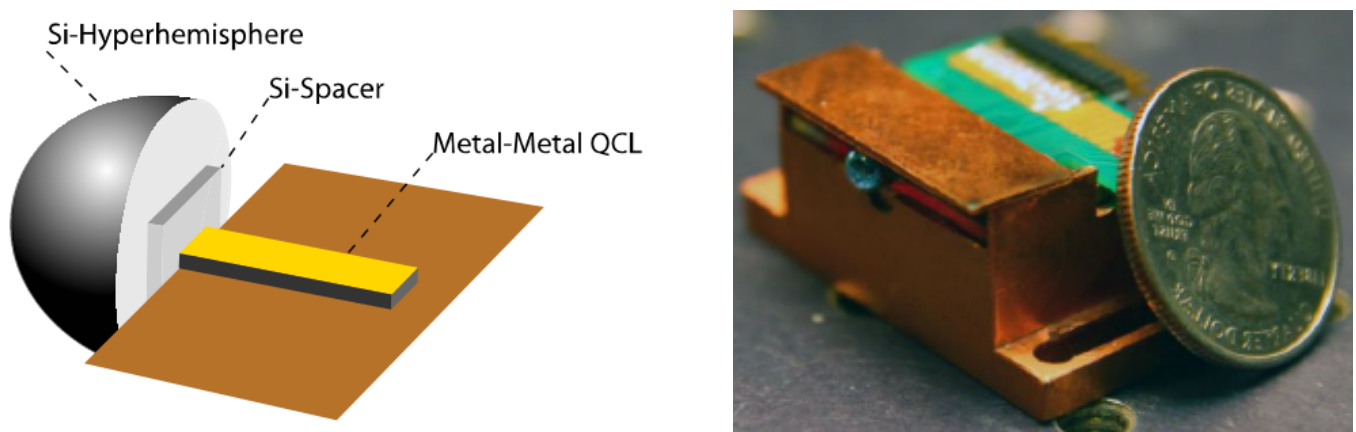

Fig.8 Lens-coupled metal-metal waveguide and packaged QCL with optical lens

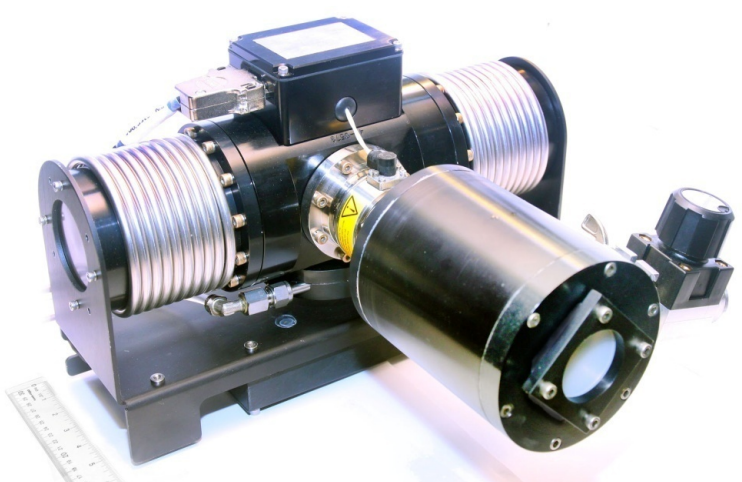

Fig.9 Stirling cycle cooler which contains the packaged QCL and cools QCL to ca. $50 \mathrm{~K}$.

\section{EASY-TO-USE QCL}

The QCL device package is mounted in the closed-cycle cryocooler (Fig.9). This cryocooler is based on the Stirling cycle, which uses two linear motors to drive two pistons 90 degrees out of phase. The pistons circulate a parcel of helium gas through a heat exchanger in a four point pressure/volume cycle. This leads to cooling of the copper cold head on which the QCL device package is attached, resulting in temperatures of $<50 \mathrm{~K}$. This product is called Easy QCL. Time averaged output power levelsof various QCLs are summarized in Fig.10. The time average power is essential for 
microbolometer THz-FPA, because it usually has thermal time constant of $10-20 \mathrm{msec}$. Duty cycle of $2 \%$ (pulse width: $200 \mathrm{nsec}$ ) is usually applied to pulsed operation, but larger value of duty cycle, such as $25 \%$ can be applied.

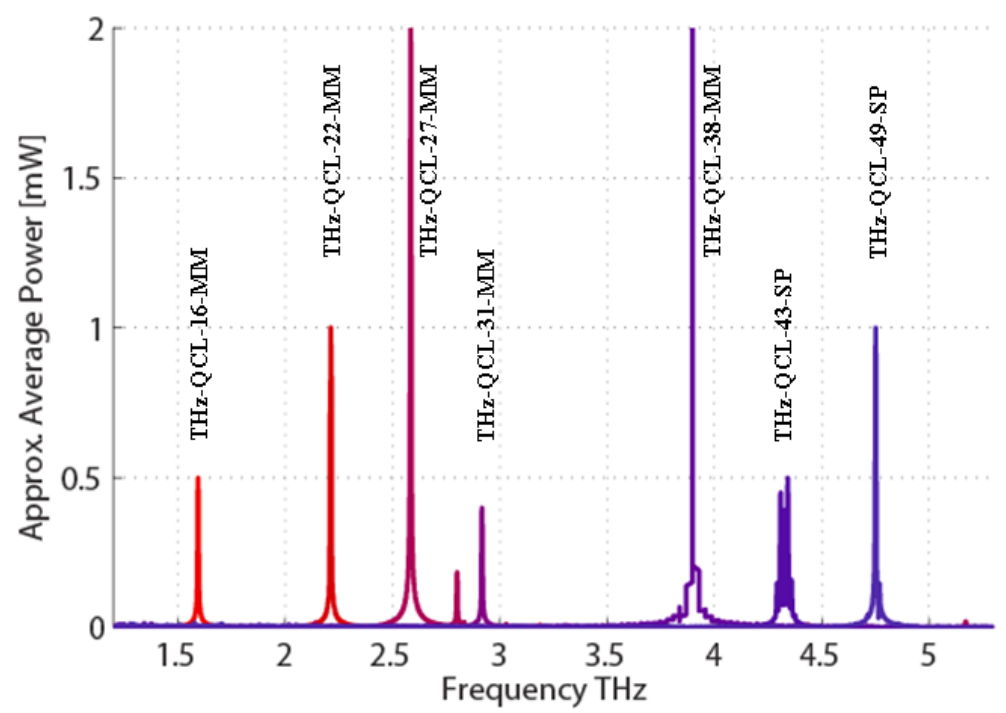

Fig.10 Frequency dependence of approximate time-average powers from QCLs (operation temperature: ca. $50 \mathrm{~K}$ )

The combined imaging equipment composed of THz evaluation camera and Easy QCL can provide real-time THz images of biomedical samples (see Fig.11), which are reaction products of lectin and glycogen without labeling molecules, deposited on hydrophobic substrate with different concentrations (the column density of the product seems low). Scales in right side of the figure show transmittance $(92-100 \%)$. The reaction products with concentrations of $25 \mu$ Molar and $5 \mu$ Molar can be seen as absorption spots at $4.2 \mathrm{THz}$. Although the experiments are preliminary, the results indicate that the combined $\mathrm{THz}$ imaging equipment composed of $\mathrm{THz}$ camera and QCL can be a good tool for label-free detection of reactions of biological molecules.

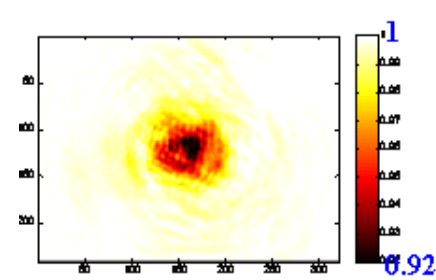

Lectin + glycogen $25 \mu \mathrm{M}$

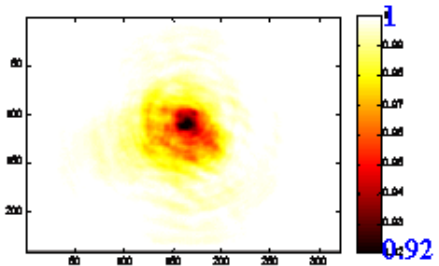

$5 \mu \mathrm{M}$

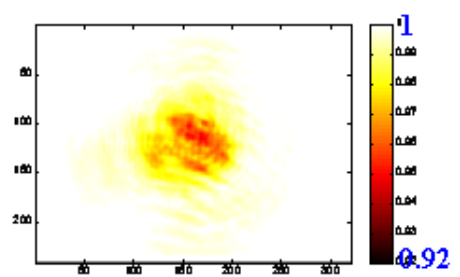

$1 \mu \mathrm{M}$

Fig.11 Real-time transmission imageries for reactions of Lectin with glycogen with different concentrations, using combined imaging equipment of Easy QCL and THz evaluation camera

\section{DESIGN OF TRANSMISSION-TYPE THz MICROSCOPE}

The concept of real-time $\mathrm{THz}$ imaging equipment, in combination of palm-size $\mathrm{THz}$ camera with Easy QCL, is shown in Fig.12. The combined equipment is transmission-type THz microscope which consists of Easy QCL, THz 
optics, sample table and palm-size THz camera. To predict the performance for the combined imaging equipment, it is assumed that sample area of $1 \mathrm{~cm}$ in diameter is uniformly irradiated by QCL and is imaged on a part of THz-FPA (5.6 $\mathrm{mm}$ in diameter, namely, ca. 45 thousand pixels), although interference patterns are, for the moment, difficult to remove. Overall transmittance of $50 \%$ is also assumed for THz optics, including package window. Figure 13 shows the predicted incident QCL power per pixel and schematic frequency dependence of NEP (Fig.2). For comparison, incident power of blackbody $(1000 \mathrm{~K})$ is also plotted. It is found that in the frequency region higher than $1.5 \mathrm{THz}$, signal-to-noise ratio (SNR) of $100-500$ can be obtained for the $1 \mathrm{~cm}$ sample area. In the case of $10 \%$ duty cycle, the SNR increases by a factor of 5. Thus, the combined imaging equipment is promising for a variety of applications. Lock-in imaging technique further improves performance by a factor of several to $10^{[19]}$.

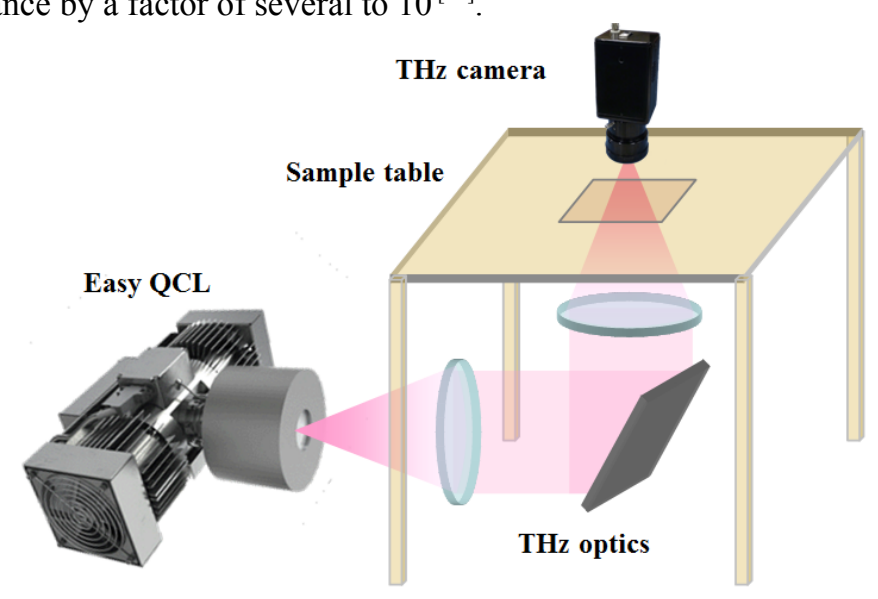

Fig.12 The concept of real-time THz imaging equipment, in combination of palm-size THz camera with Easy QCL

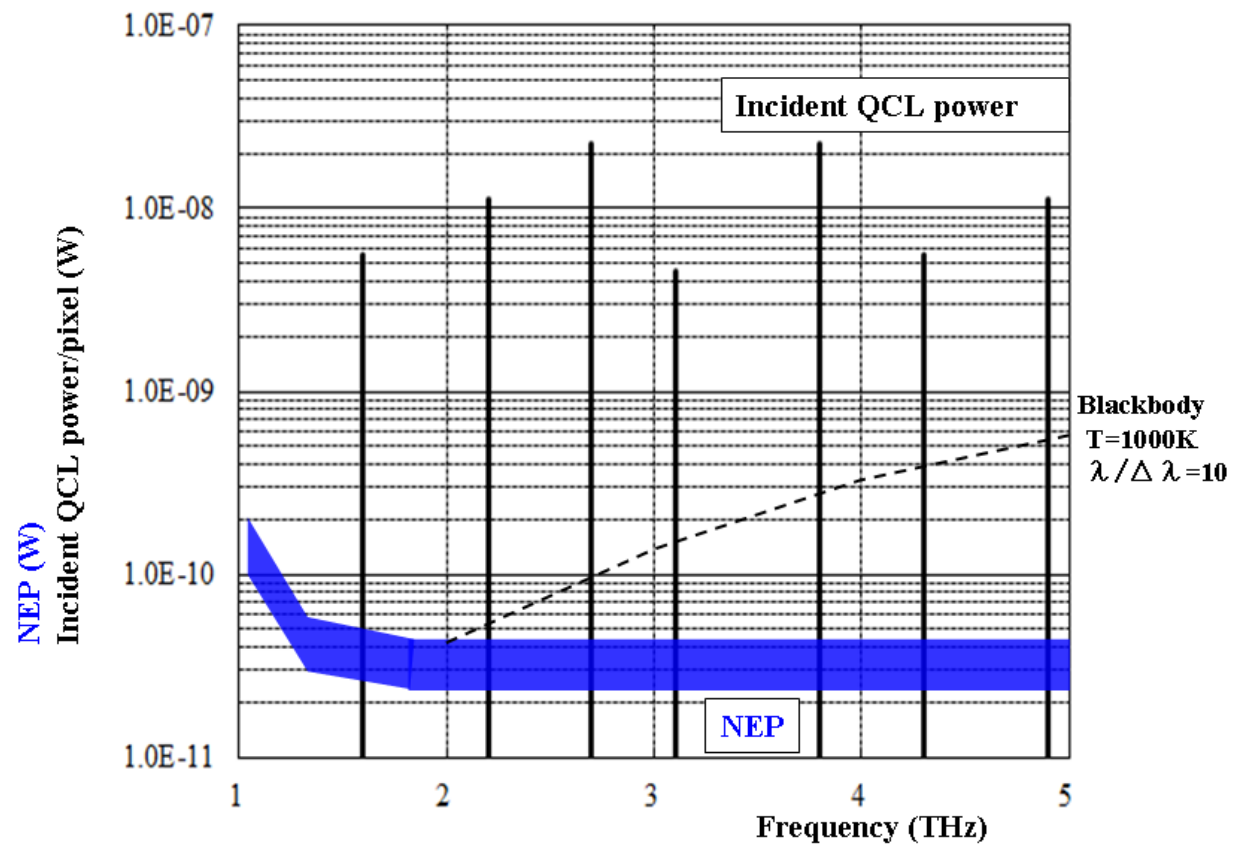

Fig.13 The predicted incident QCL power per pixel and schematic frequency dependence of NEP 


\section{SUMMARY}

Wavelength dependence of NEP for broad-band type microbolometer THz-FPA is presented, which indicates that it is achievable to establish standard method of measuring performance of $\mathrm{THz}$ detectors. The imaging experiments show that a real-time THz camera is a very efficient tool for optical alignment in complicated THz radiation source. Second, device structure of powerful compact THz-QCL is described and frequency coverage of QCL is found best-fit for the broad-band type microbolometer THz-FPA. Finally, the concept of real-time transmission-type $\mathrm{THz}$ microscope is proposed, which consists of Easy QCL, THz optics, sample table and palm-size THz camera. The performance of the $\mathrm{THz}$ microscope is predicted, i.e., signal-to-noise ratio of 100 - $500 \mathrm{can}$ be obtained for the $1 \mathrm{~cm}$ sample area.

\section{ACKNOWLEDGEMENTS}

This research was supported by the project of National Institute of Information and Communications Technology. Authors are very much grateful to Drs. H. Minamide and S. Hayashi (RIKEN), Dr. F. Destic and his colleagues (ISAE), Prof. M. Asada and his colleagues (Tokyo Institute of Technology) for making joint experiments, using their THz sources. Authors also thank Prof. M. Gonokami and his colleagues (University of Tokyo) and Dr. J. Itatani (the Institute for Solid State Physics, the Univ. of Tokyo) for providing their data, and Prof. H. Tabata and his colleagues (University of Tokyo) for preparing biological samples.

\section{REFERENCES}

[1] Ogawa, Y., Hayashi, S., Oikawa, M., Otani, C., and Kawase, K., "Interference terahertz label-free imaging for protein detection on a membrane," OPTICS EXPRESS, 16, pp.22083-22089 (2008).

[2] Brun, M-A., Formanek, F., Yasuda, A., Sekine, M., Ando, N., and Eishii, Y., “Terahertz imaging applied to cancer diagnosis,” Phys. Med. Biol., 55, pp.4615-4623 (2010) .

[3] Minamide, H., and Ito, H., "Frequency-agile terahertz-wave sources and applications to sensitive diagnosis of semiconductor wafers," Proc. SPIE, 8023, 802301 (2011).

[4] Kawase, K., "Terahertz imaging for drug detection and large-scale integrated circuit inspection,” Optics \& Photonics News, 15, pp.34-39 (2004).

[5] Hosako, I., Sekine, N., Oda, N., Sano, M., Kurashina, S., Miyoshi, M., Sonoda, K., Yoneyama, H., and Sasaki, T., “A real-time terahertz imaging system consisting of terahertz quantum cascade laser and uncooled microbolometer array detector,” Proc. SPIE, 8023, 80230A (2011).

[6] Taday, P. F., Bradley, I. V., Arnone, D. D., and Pepper, M., "Using Terahertz Pulse Spectroscopy to Study the Crystalline Structure of a Drug: A Case Study of the Polymorphs of Ranitidine Hydrochloride,” J. Pharmaceutical Sci., 92, pp.831-838 (2003).

[7] Fitzgerald, A. J., Cole, B. E., and Taday, P. F., "Nondestructive Analysis of Tablet Coating Thicknesses Using Terahertz Pulsed Imaging," J. Pharmaceutical Sci., 94, pp.177-183 (2005).

[8] Shen,Y. C., Taday, P. F., Newnham, D. A., and Pepper, M., "Chemical mapping using reflection terahertz pulsed 
imaging," Semicond. Sci. Technol. 20, pp.S254-S257 (2005).

[9] Oda, N., "Uncooled bolometer-type Terahertz focal plane array and camera for real-time imaging," Comptes Rendus Physique, 11, 496-509 (2010).

[10] Meilhan, J., Dupont, B., Goudon, V., Lasfargues, G., Lalanne Dera, J., Nguyen, D., Ouvrier-Buffet, J., Pocas, S., Maillou, T., Cathabard, O., Barbieri, S., and Simoens, F., " Active THz imaging and explosive detection with uncooled antenna-coupled microbolometer arrays," Proc. SPIE, 8023, 80230E (2011).

[11] Bolduc, M., Terroux, M., Tremblay, B., Marchese, L., Savard, E., Doucet, M., Oulachgar, H., Alain, C., Jerominek, H., and Bergeron, A.," Noise-equivalent power characterization of an uncooled microbolometer-based THz imaging camera," Proc. SPIE, 8023, 80230C (2011).

[12] Oda, N., "HANDY TERAHERTZ CAMERA FOR REAL-TIME IMAGING,” ICAS JAIMA Symposium 2011, p1 (2011).

[13] Lee, A. W. M., Williams, B. S., Kumar, S., Hu, Q., and Reno, J. L., " Real-Time Imaging Using a 4.3-THz Quantum Cascade Laser and a 320x240 Microbolometer Focal-Plane Array," IEEE PHOTONICS TECHNOLOGY LETTERS, 18, pp.1415-1417 (2006).

[14] Hosako, I., Sekine, N., Patrashin, M., Saito, S., Fukunaga, K., Kasai, Y., Baron, P., Seta, T., Mendrok, J., Ochiai, S., and Yasuda, H., “At the Dawn of a New Era in Terahertz Technology,” Proc. The IEEE, 95, pp.1611-1623 (2007).

[15] Faist, J., Capasso, F., Sivco, D. L., Sirtori, C., Hutchinson, A. L., and Cho, A. Y., "Quantum Cascade Lasers," Science, 264, pp.553-556 (1994).

[16] Köhler, R., Tredicucci, A., Beltram, F., Beere, H., Linfield, E., Davies, A., Ritchie, D., Lotti, R., and Rossi, F., “Terahertz semiconductor-heterostructure laser," Nature 417, p156 (2002).

[17] Hayashi, S., Nawata, K., Sakai, H., Taira, T., Minamide, H., and Kawase, K., "High-power, single-longitudinal-mode terahertz-wave generation pumped by a microchip Nd:YAG laser,” OPTICS EXPRESS, 20, pp. 2881 (2012).

[18] Oda, N., Yoneyama, H., Sasaki, T., Sano. M., Kurashina, S., Hosako, I., Sekine, N., Sudou, T., and Irie, T., "Detection of terahertz radiation from quantum cascade laser, using vanadium oxide microbolometer focal plane arrays," Proc. SPIE, 6940, 69402Y-1-69402Y-12 (2008).

[19] Oda, N., Sano, M., Sonoda, K., Yoneyama, H., Kurashina, S., Miyoshi, M., Sasaki, T., Hosako, I., Sekine, N., Sudou, T., and Ohkubo, S., "Development of Terahertz Focal Plane Arrays and Handy Camera," Proc. SPIE, 8012, 80121B, (2011).

[20] Tohyama, S., Miyoshi, M., Kurashina, S., Ito, I., Sasaki, T., Ajisawa, A., Tanaka, Y., Kawahara, A., Iida, K., and Oda, N., " New thermally isolated pixel structure for high resolution $(640 \times 480)$ uncooled infrared focal plane arrays," Optical Engineering, 45, 014001 (2006).

[21] Hebling, J., Almási, G., Kozma, I. Z., and Kuhl, J., "Velocity matching by pulse front tilting for large area THz-pulse generation," Opt. Express, 10, 1161(2002).

[22] Hirori, H., Doi, A., Blanchard, F., and Tanaka, K., "Single-cycle terahertz pulses with amplitudes exceeding 1 
MV/cm generated by optical rectification in $\mathrm{LiNbO}_{3}$," Appl. Phys. Lett., 98, 091106 (2011).

[23] Williams, B. S., Kumar, S., Hu, Q., and Reno, J. L., "High-power terahertz quantum-cascade lasers," Electron. Lett., 42, pp.89-90 (2006).

[24] Hu, Q., Williams, B. S., Kumar, S., Callebaut, H., Kohen, S., and Reno, J. L., "Resonant-phonon-assisted THz quantum-cascade lasers with metal-metal waveguides,” Semiconduc. Sci. Technol., 20, pp.S228-S236 (2005). 